\title{
Laplace Transform Method for Economic Models with Constant Proportional Caputo Derivative
}

\author{
Esra Karatas Akgül ${ }^{1, *}$, Ali Akgül ${ }^{1}$ and Dumitru Baleanu ${ }^{2,3,4}$ (1) \\ 1 Art and Science Faculty, Department of Mathematics, Siirt University, TR-56100 Siirt, Turkey; \\ aliakgul@siirt.edu.tr \\ Department of Mathematics, Cankaya University, Balgat, 06530 Ankara, Turkey; dumitru@cankaya.edu.tr \\ 3 Institute of Space Sciences, R76900 Magurele-Bucharest, Romania \\ 4 Department of Medical Research, China Medical University, Taichung 40402, Taiwan \\ * Correspondence: esrakaratas@siirt.edu.tr
}

Received: 30 May 2020; Accepted: 25 June 2020; Published: 3 July 2020

check for updates

\begin{abstract}
In this study, we solved the economic models based on market equilibrium with constant proportional Caputo derivative using the Laplace transform. We proved the accuracy and efficiency of the method. We constructed the relations between the solutions of the problems and bivariate Mittag-Leffler functions.
\end{abstract}

Keywords: Laplace transforms; constant proportional Caputo derivative; modeling

MSC: 44A10; 26A33

\section{Introduction}

Fractional differential equations have taken much interest in the recent years. Ghanbari et al. [1,2] investigated the new application of fractional Atangana-Baleanu derivatives and abundant new analytical and approximate solutions to the generalized Schamel equation. Allahviranloo et al. [3] worked the fuzzy fractional differential equation with Atangana-Baleanu fractional derivative approach. Salari et al. [4] searched existence and multiplicity for some boundary value problems involving Caputo and Atangana-Baleanu fractional derivatives. Akgül et al. [5-7] investigated the solutions of new type fractional differential equations occurring in the electrohydrodynamic flow, analysis and dynamical behavior of fractional-order cancer model with vaccine strategy, and the solutions of fractional order telegraph partial differential equation by Crank-Nicholson finite difference method.

We consider the following economic models:

$$
q_{d}(x)=d_{0}-d_{1} h(x), \quad q_{s}(x)=-s_{0}+s_{1} h(x),
$$

where $h$ the price of goods, $d_{0}, s_{0}, d_{1}, s_{1}$ are positive constants. For $q_{d}(x)=q_{s}(x)$, when the demanded quantity and the supplied quantity are equal, the equilibrium price is found as; $h^{*}=\frac{d_{0}+s_{0}}{d_{1}+s_{1}}$. Then, the price is disposed to stay stable. Now, considering [8]:

$$
h^{\prime}(x)=k\left(q_{d}-q_{s}\right) .
$$

where $k>0$. Then, we get

$$
h^{\prime}(x)+k\left(d_{1}+s_{1}\right) h(x)=k\left(d_{0}+s_{0}\right) .
$$


When we solved the first order ordinary differential equation, the solution can be found:

$$
h(x)=\frac{d_{0}+s_{0}}{d_{1}+s_{1}}-\left[h(0)+\frac{d_{0}+s_{0}}{d_{1}+s_{1}}\right] \exp \left(-k\left(d_{1}+s_{1}\right) x\right),
$$

where $h(0)$ is the price at the time $x=0$ and in here we do not interest to the expectation of agents in market.

In the above equation, we define the $h(0)$ as the expense at the time $x=0$. If we take into consideration the prospects of agents, the request and provision functions containing supplement elements $q_{d}$ and $q_{d}$ alters as:

$$
q_{d}(x)=d_{0}-d_{1} h(x)+d_{2} h^{\prime}(x), \quad q_{s}(x)=-s_{0}+s_{1} h(x)-s_{2} h^{\prime}(x) .
$$

We equalize $q_{d}(x), q_{s}(x)$ and obtain:

$$
h^{\prime}(x)-\frac{d_{1}+s_{1}}{d_{2}+s_{2}} h(x)=-\frac{d_{0}+s_{0}}{d_{2}+s_{2}} .
$$

and when the linear differential Equation (6) is solved, the solution is acquired as:

$$
h(x)=\frac{d_{0}+s_{0}}{d_{1}+s_{1}}-\left[h(0)+\frac{d_{0}+s_{0}}{d_{1}+s_{1}}\right] \exp \left(\frac{d_{1}+s_{1}}{d_{2}+s_{2}} x\right) .
$$

We consider the above economic models with constant proportional Caputo derivative that has been presented very recently in [9]. We construct the Laplace transform method (LTM) to solve the economic models. The Laplace transform is one of the best integral transform used by many researchers.

Gupta et al. [10] investigated analytical solutions of convection-diffusion problems by combining Laplace transform method and homotopy perturbation method. Anjum et al. [11] worked Laplace transform making the variational iteration method easier. Convergence of iterative Laplace transform methods for a system of fractional partial differential equations and partial integro-ifferential equations arising in option pricing has been searched by Zhou [12]. Bashir et al. [13] studied solution of non-homogeneous differential equations using Faddeev-Leverrier method together with Laplace transform. Convergence analysis of iterative Laplace transform methods for the coupled partial differential equations from regime-switching option pricing was investigated by Jingtang [14]. Eljaoui et al. [15] researched Aumann fuzzy improper integral and its application to solve fuzzy integro-differential equations by LTM. Zhou et al. [16] have worked fast LTM for free-boundary problems of fractional diffusion equations. The transform method for the ulam stability of linear fractional differential equations with constant coefficient was studied by Yonghong [17]. Fatoorehchi et al. [18] investigated series solution of nonlinear differential equations by a novel extension of the LTM. Jacobs [19] searched high-order compact finite difference and Laplace transform method for the solution of time-fractional heat equations with Dirichlet and Neumann boundary conditions. See these reference for more details [20,21].

Mittag-Leffler functions are very important in the fractional calculus. There are many useful works related to the Mittag-Leffler functions in the literature. Özarslan et al. [22,23] studied on a singular integral equation including a set of multivariate polynomials suggested by Laguerre polynomials and on a certain bivariate Mittag-Leffler function analyzed from a fractional-calculus point of view. For more details [24-29].

Acay et al. [30] found the solutions of these models with three different derivatives which are Caputo, Caputo-Fabrizio and Atangana-Baleanu. They presented the efficiency of the Laplace transform method for these models. For more details see [31-33].

We organize the paper as follows: we give the main definitions and lemmas related to the constant proportional Caputo derivative in Section 2. We present the applications of the economic models by Laplace transform method in Section 3. We give some conclusions remarks in the last section. 


\section{Preliminaries}

Definition 1. The Caputo fractional derivative is defined as [34]:

$$
{ }_{a}^{C} D^{\sigma} h(x)=\frac{1}{\Gamma(1-\sigma)} \int_{a}^{x}(x-\tau)^{-\sigma} h^{\prime}(\tau) d \tau,
$$

where $0<\sigma \leq 1$.

Definition 2. The Riemann-Liouville integral is given as [34];

$$
{ }_{a}^{R L} I^{\sigma} h(x)=\frac{1}{\Gamma(\sigma)} \int_{a}^{x}(x-\tau)^{\sigma-1} h(\tau) d \tau .
$$

in here $h$ is an integrable function and $\sigma>0$.

From above definitons,

$$
{ }_{a}^{C} D^{\sigma} h(x)={ }_{a}^{R L} I^{1-\sigma} h^{\prime}(x) .
$$

is written.

Definition 3. The constant proportional Caputo (CPC) derivative is defined by [9]

$$
{ }_{0}^{C P C} D_{x}^{\alpha} h(x)=\frac{1}{\Gamma(1-\alpha)} \int_{0}^{x}\left[k_{1}(\alpha) h(\tau)+k_{0}(\alpha) h^{\prime}(\tau)\right](x-\tau)^{-\alpha} d \tau .
$$

Definition 4. Let $\xi, \eta:[0, \infty) \rightarrow \mathfrak{R}$, then the convolution of $\xi, \eta$ is

$$
(\xi * \eta)=\int_{0}^{x} \xi(x-u) \eta(u) d u .
$$

and assume that $\xi, \eta:[0, \infty) \rightarrow \Re$, then we write:

$$
L\{(\xi * \eta)(x)\}=L\{\xi(x)\} L\{\eta(x)\}
$$

Definition 5. We define $E_{\alpha}(v)$ by:

$$
E_{\alpha}(v)=\sum_{l=0}^{\infty} \frac{v^{l}}{\Gamma(\alpha l+1)} \quad(v \in \mathbb{C}, \operatorname{Re}(\alpha)>0) .
$$

The Mittag-Leffler function which has two parameters is given as:

$$
E_{\alpha, \beta}(v)=\sum_{l=0}^{\infty} \frac{v^{l}}{\Gamma(\alpha l+\beta)} \quad(v, \beta \in \mathbb{C}, \operatorname{Re}(\alpha)>0),
$$

it is worth, $E_{\alpha, \beta}(v)$ corresponds to the Mittag-Leffler function (14) when $\beta=1$.

Lemma 1. The Laplace transform of constant proportional Caputo (CPC) derivative is found as [9], for Laplace transform of the derivatives see Table 1:

$$
L\left\{{ }_{0}^{C P C} D_{x}^{\alpha} h(x)\right\}=\left[\frac{k_{1}(\alpha)}{s}+k_{0}(\alpha)\right] s^{\alpha} L\{h(x)\}-k_{0}(\alpha) s^{\alpha-1} h(0) .
$$


Table 1. Laplace transform of the Caputo (C), constant proportional Caputo (CPC) derivatives and the Riemann-Liouville (RL) integral.

\begin{tabular}{lll}
\hline C, CPC and RL & Convolution & Laplace Transform \\
\hline${ }_{0}^{C} D_{t}^{\alpha} f(t)$ & $\frac{d f(t)}{d t} * \frac{t^{-\alpha}}{\Gamma(1-\alpha)}$ & $(s L\{f(t)\}-f(0)) s^{\alpha-1}$ \\
\hline${ }_{0}^{R L} I_{t}^{\alpha} f(t)$ & $f(t) * \frac{t^{\alpha-1}}{\Gamma(\alpha)}$ & $s^{-\alpha} L\{f(t)\}$ \\
\hline${ }_{0}^{C P C} D_{t}^{\alpha} f(t)$ & $f(t) * \frac{k_{1}(\alpha) t^{-\alpha}}{\Gamma(1-\alpha)}+\frac{d f(t)}{d t} * \frac{k_{0}(\alpha) t^{-\alpha}}{\Gamma(1-\alpha)}$ & $k_{1}(\alpha) s^{\alpha-1} L\{f(t)\}+(s L\{f(t)\}-f(0)) s^{\alpha-1} k_{0}(\alpha)$ \\
\hline
\end{tabular}

\section{Applications of the Economic Model}

Let us take into consideration the first model with the constant proportional Caputo derivative as:

$$
{ }_{0}^{C P C} D_{x}^{\alpha} h(x)+k\left(d_{1}+s_{1}\right) h(x)=k\left(d_{0}+s_{0}\right) .
$$

Applying the Laplace transform to the equation, we acquire

$$
L\left\{\begin{array}{l}
C P C \\
0
\end{array} D_{x}^{\alpha} h(x)\right\}+k\left(d_{1}+s_{1}\right) L\{h(x)\}=L\left\{k\left(d_{0}+s_{0}\right)\right\} .
$$

Using the expression Lemma 1, then we obtain:

$$
\left[\frac{k_{1}(\alpha)}{s}+k_{0}(\alpha)\right] s^{\alpha} L\{h(x)\}-k_{0}(\alpha) s^{\alpha-1} h(0)+k\left(d_{1}+s_{1}\right) L\{h(x)\}=\frac{k\left(d_{0}+s_{0}\right)}{s},
$$

and

$$
\begin{aligned}
& L\{h(x)\}=\frac{k\left(d_{0}+s_{0}\right)}{k_{1}(\alpha) s^{\alpha}+k_{0}(\alpha) s^{\alpha+1}+k\left(d_{1}+s_{1}\right) s} \\
& +\frac{k_{0}(\alpha) h(0) s^{\alpha}}{k_{1}(\alpha) s^{\alpha}+k_{0}(\alpha) s^{\alpha+1}+k\left(d_{1}+s_{1}\right) s} \\
& =\frac{d_{0}+s_{0}}{d_{1}+s_{1}} s^{-1}\left[1-\frac{-k_{1}(\alpha) s^{\alpha-1}-k_{0} \alpha s^{\alpha}}{k\left(d_{0}+s_{0}\right)}\right]^{-1} \\
& +h(0) s^{-1}\left[1-\frac{-k_{1}(\alpha) s^{-1}-k\left(d_{1}+s_{1}\right) s^{-\alpha}}{k_{0}(\alpha)}\right]^{-1} \\
& =\frac{d_{0}+s_{0}}{d_{1}+s_{1}} s^{-1} \sum_{j=0}^{\infty}\left[\frac{-k_{1}(\alpha) s^{\alpha-1}-k_{0}(\alpha) s^{\alpha}}{k\left(d_{0}+s_{0}\right)}\right]^{j} \\
& +h(0) s^{-1} \sum_{j=0}^{\infty}\left[\frac{-k_{1}(\alpha) s^{-1}-k\left(d_{1}+s_{1}\right) s^{-\alpha}}{k_{0}(\alpha)}\right]^{j} \\
& =\frac{d_{0}+s_{0}}{d_{1}+s_{1}} s^{-1} \sum_{j=0}^{\infty} \frac{1}{k^{j}\left(d_{0}+s_{0}\right)^{j}} \sum_{r=0}^{j}\left(\begin{array}{l}
j \\
r
\end{array}\right)\left[-k_{1}(\alpha) s^{\alpha-1}\right]^{j-r}\left[-k_{0}(\alpha) s^{\alpha}\right]^{r} \\
& +h(0) s^{-1} \sum_{j=0}^{\infty} \frac{1}{k_{0}(\alpha)^{j}} \sum_{r=0}^{j}\left(\begin{array}{l}
j \\
r
\end{array}\right)\left[-k_{1}(\alpha) s^{-1}\right]^{j-r}\left[-k\left(d_{1}+s_{1}\right) s^{-\alpha}\right]^{r} \\
& =\frac{d_{0}+s_{0}}{d_{1}+s_{1}} \sum_{j=0}^{\infty} \sum_{r=0}^{j}(-1)^{j} \frac{k_{1}(\alpha)^{j-r} k_{0}(\alpha)^{r}}{k^{j}\left(d_{0}+s_{0}\right)^{j}}\left(\begin{array}{l}
j \\
r
\end{array}\right) s^{(\alpha-1)(j-r)+\alpha r-1} \\
& +h(0) \sum_{j=0}^{\infty} \sum_{r=0}^{j}(-1)^{j} \frac{k_{1}(\alpha)^{j-r} k^{r}\left(d_{1}+s_{1}\right)^{r}}{k_{0}(\alpha)^{j}}\left(\begin{array}{l}
j \\
r
\end{array}\right) s^{j-r-\alpha r-1} .
\end{aligned}
$$


Then, implementing the inverse LTM gives:

$$
\begin{aligned}
h(x)= & \frac{d_{0}+s_{0}}{d_{1}+s_{1}} \sum_{j=0}^{\infty} \sum_{r=0}^{j}(-1)^{j} \frac{k_{1}(\alpha)^{j-r} k_{0}(\alpha)^{r}}{k^{j}\left(d_{0}+s_{0}\right)^{j}}\left(\begin{array}{l}
j \\
r
\end{array}\right) \frac{x^{(1-\alpha) j-r}}{\Gamma((1-\alpha) j-r+1)} \\
& +h(0) \sum_{j=0}^{\infty} \sum_{r=0}^{j}(-1)^{j} \frac{k_{1}(\alpha)^{j-r} k^{r}\left(d_{1}+s_{1}\right)^{r}}{k_{0}(\alpha)^{j}}\left(\begin{array}{l}
j \\
r
\end{array}\right) \frac{x^{j+(\alpha-1) r}}{\Gamma(j+(\alpha-1) r+1)},
\end{aligned}
$$

When we take $p=j-r$, we will get:

$$
\begin{aligned}
h(x)= & \frac{d_{0}+s_{0}}{d_{1}+s_{1}} \sum_{r=0}^{\infty} \sum_{p=0}^{\infty} \frac{(r+p) !}{r ! p !} \frac{\left(-k_{1}(\alpha)\right)^{p}\left(-k_{0}(\alpha)\right)^{r}}{k^{p+r}\left(d_{0}+s_{0}\right)^{p+r}} \frac{x^{(1-\alpha) p-\alpha r}}{\Gamma((1-\alpha) p-\alpha r+1)} \\
& +h(0) \sum_{r=0}^{\infty} \sum_{p=0}^{\infty} \frac{(r+p) !}{r ! p !} \frac{\left(-k_{1}(\alpha)\right)^{p}\left(-k\left(d_{1}+s_{1}\right)\right)^{r}}{k_{0}(\alpha)^{p+r}} \frac{x^{p+\alpha r}}{\Gamma(p+\alpha r+1)} \\
h(x)= & \frac{d_{0}+s_{0}}{d_{1}+s_{1}} \sum_{r=0}^{\infty} \sum_{p=0}^{\infty} \frac{(r+p) !}{r ! p !}\left[\frac{-k_{0}(\alpha)}{k\left(d_{0}+s_{0}\right)} x^{-\alpha}\right]^{r}\left[\frac{-k_{1}(\alpha)}{k\left(d_{0}+s_{0}\right)} x^{1-\alpha}\right]^{p} \frac{1}{\Gamma((1-\alpha) p-\alpha r+1)} \\
& +h(0) \sum_{r=0}^{\infty} \sum_{p=0}^{\infty} \frac{(r+p) !}{r ! p !}\left[\frac{-k\left(d_{1}+s_{1}\right)}{k_{0}(\alpha)} x^{\alpha}\right]^{r}\left[\frac{-k_{1}(\alpha)}{k_{0}(\alpha)} x\right]^{p} \frac{1}{\Gamma(p+\alpha r+1)} .
\end{aligned}
$$

We can write geometric series as [35]:

$$
\begin{aligned}
h(x)= & \frac{d_{0}+s_{0}}{d_{1}+s_{1}} E_{1-\alpha,-\alpha, 1}^{1}\left(\frac{-k_{1}(\alpha)}{k\left(d_{0}+s_{0}\right)} x^{1-\alpha}, \frac{-k_{0}(\alpha)}{k\left(d_{0}+s_{0}\right)} x^{-\alpha}\right) \\
& +h(0) E_{1, \alpha, 1}^{1}\left(\frac{-k_{1}(\alpha)}{k_{0}(\alpha)} x, \frac{-k\left(d_{1}+s_{1}\right)}{k_{0}(\alpha)} x^{\alpha}\right) .
\end{aligned}
$$

Let us take into consideration the second model with the constant proportional Caputo derivative as:

$$
{ }_{0}^{C P C} D_{x}^{\alpha} h(x)-\frac{d_{1}+s_{1}}{d_{2}+s_{2}} h(x)=-\frac{d_{0}+s_{0}}{d_{2}+s_{2}} .
$$

If we implement the LTM, we will get

$$
L\left\{{ }_{0}^{C P C} D_{x}^{\alpha} h(x)\right\}+k\left(d_{1}+s_{1}\right) L\{h(x)\}=L\left\{k\left(d_{0}+s_{0}\right)\right\} .
$$

Using the expression Lemma 1, then we obtain:

$$
\left[\frac{k_{1}(\alpha)}{s}+k_{0}(\alpha)\right] s^{\alpha} L\{h(x)\}-k_{0}(\alpha) s^{\alpha-1} h(0)-\frac{d_{1}+s_{1}}{d_{2}+s_{2}} L\{h(x)\}=-\frac{d_{0}+s_{0}}{d_{2}+s_{2} s},
$$


and

$$
\begin{aligned}
L\{h(x)\}= & -\frac{d_{0}+s_{0}}{d_{2}+s_{2} k_{1}(\alpha) s^{\alpha}+k_{0}(\alpha)\left(d_{2}+s_{2}\right) s^{\alpha+1}-\left(d_{1}+s_{1}\right) s} \\
& +\frac{k_{0}(\alpha) h(0) s^{\alpha}\left(d_{2}+s_{2}\right)}{\left(d_{2}+s_{2}\right) k_{1}(\alpha) s^{\alpha}+k_{0}(\alpha)\left(d_{2}+s_{2}\right) s^{\alpha+1}-\left(d_{1}+s_{1}\right) s} \\
= & \frac{d_{0}+s_{0}}{d_{1}+s_{1}} s^{-1}\left[1-\frac{\left(d_{2}+s_{2}\right) s^{\alpha-1}+k_{0} \alpha s^{\alpha}}{\left(d_{1}+s_{1}\right)}\right]^{-1} \\
& +h(0) s^{-1}\left[1-\frac{-k_{1}(\alpha)\left(d_{2}+s_{2}\right) s^{-1}+\left(d_{1}+s_{1}\right) s^{-\alpha}}{k_{0}(\alpha)\left(d_{2}+s_{2}\right)}\right]^{-1} \\
= & \frac{d_{0}+s_{0}}{d_{1}+s_{1}} s^{-1} \sum_{j=0}^{\infty}\left[\frac{\left(d_{2}+s_{2}\right) s^{\alpha-1}+k_{0}(\alpha)\left(d_{2}+s_{2}\right) s^{\alpha}}{\left(d_{1}+s_{1}\right)}\right]^{j} \\
& +h(0) s^{-1} \sum_{j=0}^{\infty}\left[\frac{-k_{1}(\alpha)\left(d_{2}+s_{2}\right) s^{-1}+\left(d_{1}+s_{1}\right) s^{-\alpha}}{k_{0}(\alpha)\left(d_{2}+s_{2}\right)}\right]^{j} \\
= & \frac{d_{0}+s_{0}}{d_{1}+s_{1}} s^{-1} \sum_{j=0}^{\infty} \frac{1}{\left(d_{1}+s_{1}\right)^{j} \sum_{r=0}^{j}\left(\begin{array}{l}
j \\
r
\end{array}\right)\left[\left(d_{2}+s_{2}\right) s^{\alpha-1}\right]^{j-r}\left[k_{0}(\alpha)\left(d_{2}+s_{2}\right) s^{\alpha}\right]^{r}} \\
& +h(0) s^{-1} \sum_{j=0}^{\infty} \frac{1}{k_{0}(\alpha)^{j}\left(d_{2}+s_{2}\right)^{j}} \sum_{r=0}^{j}\left(\begin{array}{l}
j \\
r
\end{array}\right)\left[-k_{1}(\alpha)\left(d_{2}+s_{2}\right) s^{-1}\right]^{j-r}\left[\left(d_{1}+s_{1}\right) s^{-\alpha}\right]^{r} \\
= & \frac{d_{0}+s_{0}}{d_{1}+s_{1}} \sum_{j=0}^{\infty} \sum_{r=0}^{j} \frac{\left(d_{2}+s_{2}\right)^{j-r} k_{0}(\alpha)^{r}\left(d_{2}+s_{2}\right)^{r}}{\left(d_{1}+s_{1}\right)^{j}}\left(\begin{array}{l}
j \\
r
\end{array}\right) s^{(\alpha-1)(j-r)+\alpha r-1} \\
& +h(0) \sum_{j=0}^{\infty} \sum_{r=0}^{j} \frac{\left(-k_{1}(\alpha)\right)^{j-r}\left(d_{2}+s_{2}\right)^{j-r}\left(d_{1}+s_{1}\right)^{r}}{k_{0}(\alpha)^{j}\left(d_{2}+s_{2}\right)^{j}}\left(\begin{array}{l}
j \\
r
\end{array}\right) s^{r-j-\alpha r-1} .
\end{aligned}
$$

Applying the inverse Laplace transform, we have:

$$
\begin{aligned}
h(x)= & \frac{d_{0}+s_{0}}{d_{1}+s_{1}} \sum_{j=0}^{\infty} \sum_{r=0}^{j} \frac{\left(d_{2}+s_{2}\right)^{j-r} k_{0}(\alpha)^{r}\left(d_{2}+s_{2}\right)^{r}}{\left(d_{1}+s_{1}\right)^{j}}\left(\begin{array}{l}
j \\
r
\end{array}\right) \frac{x^{(1-\alpha)(j-r)-\alpha r}}{\Gamma((1-\alpha)(j-r)-\alpha r+1)} \\
& +h(0) \sum_{j=0}^{\infty} \sum_{r=0}^{j} \frac{\left(-k_{1}(\alpha)\right)^{j-r}\left(d_{2}+s_{2}\right)^{j-r}\left(d_{1}+s_{1}\right)^{r}}{k_{0}(\alpha)^{j}\left(d_{2}+s_{2}\right)^{j}}\left(\begin{array}{l}
j \\
r
\end{array}\right) \frac{x^{j-r+\alpha r}}{\Gamma((j-r+\alpha r+1)} .
\end{aligned}
$$

When we take $p=j-r$, we will get:

$$
\begin{aligned}
h(x)= & \frac{d_{0}+s_{0}}{d_{1}+s_{1}} \sum_{r=0}^{\infty} \sum_{p=0}^{\infty} \frac{(r+p) !}{r ! p !} \frac{\left(\left(d_{2}+s_{2}\right)\right)^{p+m}\left(k_{0}(\alpha)\right)^{r}}{\left(d_{1}+s_{1}\right)^{p+r}} \frac{x^{(1-\alpha) p-\alpha r}}{\Gamma((1-\alpha) p-\alpha r+1)} \\
& +h(0) \sum_{r=0}^{\infty} \sum_{p=0}^{\infty} \frac{(r+p) !}{r ! p !} \frac{\left(-k_{1}(\alpha)\right)^{p}\left(\left(d_{1}+s_{1}\right)\right)^{r}}{k_{0}(\alpha)^{p+r}}\left(d_{2}+s_{2}\right)^{r} \frac{x^{p+\alpha r}}{\Gamma(p+\alpha r+1)} \\
h(x)= & \frac{d_{0}+s_{0}}{d_{1}+s_{1}} \sum_{r=0}^{\infty} \sum_{p=0}^{\infty} \frac{(r+p) !}{r ! p !}\left[\frac{\left(d_{2}+s_{2}\right) k_{0}(\alpha)}{\left(d_{1}+s_{1}\right)} x^{-\alpha}\right]^{r}\left[\frac{d_{2}+s_{2}}{d_{1}+s_{1}} x^{1-\alpha}\right]^{p} \frac{1}{\Gamma((1-\alpha) p-\alpha r+1)} \\
& +h(0) \sum_{r=0}^{\infty} \sum_{p=0}^{\infty} \frac{(r+p) !}{r ! p !}\left[\frac{\left(d_{1}+s_{1}\right)}{k_{0}(\alpha)\left(d_{2}+s_{2}\right)} x^{\alpha}\right]^{r}\left[\frac{-k_{1}(\alpha)}{k_{0}(\alpha)} x\right]^{p} \frac{1}{\Gamma(p+\alpha r+1)} .
\end{aligned}
$$


We can write geometric series as [35]:

$$
\begin{aligned}
h(x)= & \frac{d_{0}+s_{0}}{d_{1}+s_{1}} E_{1-\alpha,-\alpha, 1}^{1}\left(\frac{d_{2}+s_{2}}{d_{1}+s_{1}} x^{1-\alpha}, \frac{\left(d_{2}+s_{2}\right) k_{0}(\alpha)}{\left(d_{1}+s_{1}\right)} x^{-\alpha}\right) \\
& +h(0) E_{1, \alpha, 1}^{1}\left(\frac{-k_{1}(\alpha)}{k_{0}(\alpha)} x, \frac{\left(d_{1}+s_{1}\right)}{k_{0}(\alpha)\left(d_{2}+s_{2}\right)} x^{\alpha}\right) .
\end{aligned}
$$

\section{Comparison}

In this section, we give the results that were found for the first economic model with Caputo fractional derivative [30], Caputo-Fabrizio derivative in Caputo sense [30], Atangana-Baleanu derivative [30] and constant proportional Caputo derivative.

$$
\begin{aligned}
& h(x)=\frac{\left(d_{0}+s_{0}\right)}{\left(d_{1}+s_{1}\right)}\left[1-E_{\alpha}\left(-k\left(d_{1}+s_{1}\right) x^{\alpha}\right)\right]+h(0) E_{\alpha}\left(-k\left(d_{1}+s_{1}\right) x^{\alpha}\right), \\
& h(x)=\frac{M(\alpha) h(0) \exp \left(\frac{\alpha k\left(d_{1}+s_{1}\right) x}{-M(\alpha)+(\alpha-1) k\left(d_{1}+s_{1}\right)}\right)}{M(\alpha)-(\alpha-1) k\left(d_{1}+s_{1}\right)} \\
& -\frac{M(\alpha)\left(d_{0}+s_{0}\right)\left(-1+\exp \left(\frac{\alpha k\left(d_{1}+s_{1}\right) x}{-M(\alpha)+(\alpha-1) k\left(d_{1}+s_{1}\right)}\right)\right)+(\alpha-1) k\left(d_{1}+s_{1}\right)}{\left(d_{1}+s_{1}\right)\left(-M(\alpha)+(\alpha-1) k\left(d_{1}+s_{1}\right)\right)} \\
& h(x)=\frac{A B(\alpha) h(0)}{A B(\alpha)+(1-\alpha) k\left(d_{1}+s_{1}\right)} E_{\alpha}\left(-\frac{\alpha k\left(d_{1}+s_{1}\right) x^{\alpha}}{A B(\alpha)+(1-\alpha) k\left(d_{1}+s_{1}\right)}\right) \\
& +\frac{(1-\alpha) k\left(d_{0}+s_{0}\right)}{A B(\alpha)+(1-\alpha) k\left(d_{1}+s_{1}\right)} E_{\alpha}\left(-\frac{\alpha k\left(d_{1}+s_{1}\right) x^{\alpha}}{A B(\alpha)+(1-\alpha) k\left(d_{1}+s_{1}\right)}\right) \\
& +\frac{\left(d_{0}+s_{0}\right)}{\left(d_{1}+s_{1}\right)}\left[1-E_{\alpha}\left(-\frac{\alpha k\left(d_{1}+s_{1}\right) x^{\alpha}}{A B(\alpha)+(1-\alpha) k\left(d_{1}+s_{1}\right)}\right)\right] \\
& h(x)=\frac{\left(d_{0}+s_{0}\right)}{\left(d_{1}+s_{1}\right)} E_{1-\alpha,-\alpha, 1}^{1}\left(\frac{-k_{1}(\alpha)}{k\left(d_{0}+s_{0}\right)} x^{1-\alpha}, \frac{-k_{0}(\alpha)}{k\left(d_{0}+s_{0}\right)} x^{-\alpha}\right) \\
& +h(0) E_{1, \alpha, 1}^{1}\left(\frac{-k_{1}(\alpha)}{k_{0}(\alpha)} x, \frac{-k\left(d_{1}+s_{1}\right)}{k_{0}(\alpha)} x^{\alpha}\right) \text {. }
\end{aligned}
$$

We give the results that were found for the second economic model with Caputo fractional derivative [30], Caputo-Fabrizio derivative in Caputo sense [30], Atangana-Baleanu derivative [30] and constant proportional Caputo derivative respectively as:

$$
\begin{gathered}
h(x)=-\frac{\left(d_{0}+s_{0}\right)}{\left(d_{1}+s_{1}\right)}\left[1-E_{\alpha}\left(\frac{\left(d_{1}+s_{1}\right)}{\left(d_{2}+s_{2}\right)} x^{\alpha}\right)\right]+h(0) E_{\alpha}\left(\frac{\left(d_{1}+s_{1}\right)}{\left(d_{2}+s_{2}\right)} x^{\alpha}\right), \\
h(x)=\frac{M(\alpha) h(0)\left(d_{2}+s_{2}\right)}{M(\alpha)\left(d_{2}+s_{2}\right)+(\alpha-1) d_{1}+(\alpha-1) s_{1}} \exp \left(\frac{\alpha\left(d_{1}+s_{1}\right) x}{M(\alpha)\left(d_{2}+s_{2}\right)+(\alpha-1) d_{1}+(\alpha-1) s_{1}}\right) \\
+\frac{\left(d_{0}+s_{0}\right)\left((\alpha-1) d_{1}+(\alpha-1) s_{1}-M(\alpha)\left(-1+\exp \left(\frac{\alpha k\left(d_{1}+s_{1}\right) x}{M(\alpha)\left(d_{2}+s_{2}\right)+(\alpha-1) d_{1}+(\alpha-1) s_{1}}\right)\left(d_{2}+s_{2}\right)\right)\right)}{\left(d_{1}+s_{1}\right)\left(M(\alpha)\left(d_{2}+s_{2}\right)+(\alpha-1) d_{1}+(\alpha-1) s_{1}\right.}
\end{gathered}
$$




$$
\begin{array}{r}
h(x)=\frac{A B(\alpha) h(0)\left(d_{2}+s_{2}\right)}{A B(\alpha)\left(d_{2}+s_{2}\right)+(\alpha-1) d_{1}+(\alpha-1) s_{1}} E_{\alpha}\left(\frac{\alpha\left(d_{1}+s_{1}\right) x^{\alpha}}{A B(\alpha)\left(d_{2}+s_{2}\right)+(\alpha-1) d_{1}+(\alpha-1) s_{1}}\right) \\
-\frac{(\alpha-1)\left(d_{0}+s_{0}\right)}{A B(\alpha)\left(d_{2}+s_{2}\right)+(\alpha-1) d_{1}+(\alpha-1) s_{1}} E_{\alpha}\left(\frac{\alpha\left(d_{1}+s_{1}\right) x^{\alpha}}{A B(\alpha)\left(d_{2}+s_{2}\right)+(\alpha-1) d_{1}+(\alpha-1) s_{1}}\right) \\
+\frac{(\alpha-1)\left(d_{0}+s_{0}\right)}{\alpha\left(d_{1}+s_{1}\right)}\left[1-E_{\alpha}\left(\frac{\alpha\left(d_{1}+s_{1}\right) x^{\alpha}}{A B(\alpha)\left(d_{2}+s_{2}\right)+(\alpha-1) d_{1}+(\alpha-1) s_{1}}\right)\right] \\
h(x)=\frac{\left(d_{0}+s_{0}\right)}{\left(d_{1}+s_{1}\right)} E_{1-\alpha,-\alpha, 1}^{1}\left(\frac{\left(d_{2}+s_{2}\right)}{\left(d_{1}+s_{1}\right)} x^{1-\alpha}, \frac{\left(d_{2}+s_{2}\right) k_{0}(\alpha)}{\left(d_{1}+s_{1}\right)} x^{-\alpha}\right) \\
+h(0) E_{1, \alpha, 1}^{1}\left(\frac{-k_{1}(\alpha)}{k_{0}(\alpha)} x, \frac{\left(d_{1}+s_{1}\right)}{k_{0}(\alpha)\left(d_{2}+s_{2}\right)} x^{\alpha}\right) .
\end{array}
$$

\section{Conclusions}

In this study, we gave some details of the Laplace transform. Then, we constructed the economic models and we solved these models by the Laplace transform. We concluded that the Laplace transform is very effective for solving such problems. We presented an application of the economic models by using the newly introduced constant proportional Caputo derivative. The obtained results will be useful for researchers who interest the economic models and the integral transforms.

Author Contributions: Conceptualization, A.A. and E.K.A.; methodology, D.B.; software, A.A.; validation, A.A., E.K.A. and D.B.; formal analysis, E.K.A.; investigation, A.A.; resources, E.K.A.; data curation, D.B.; writing-original draft preparation, E.K.A.; writing—review and editing, A.A.; visualization, E.K.A.; supervision, D.B.; project administration, E.K.A.; funding acquisition, A.A. All authors have read and agreed to the published version of the manuscript.

Funding: This research received no external funding.

Conflicts of Interest: The authors declare that they do not have any conflict of interest.

\section{References}

1. Ghanbari, B.; Atangana, A. A new application of fractional Atangana-Baleanu derivatives: Designing ABC-fractional masks in image processing. Phys. Stat. Mech. Its Appl. 2020, 542, 123516, [CrossRef]

2. Ghanbari, B.; Akgül, A. Abundant new analytical and approximate solutions to the generalized Schamel equation. Phys. Scr. 2020, 95, 075201. [CrossRef]

3. Allahviranloo, T.; Ghanbari, B. On the fuzzy fractional differential equation with interval Atangana-Baleanu fractional derivative approach. Chaos Solitons Fractals 2020, 128, 109397. [CrossRef]

4. Salari, A.; Ghanbari, B. Existence and multiplicity for some boundary value problems involving Caputo and Atangana-Baleanu fractional derivatives: A variational approach. Chaos Solitons Fractals 2019, 127, 312-317. [CrossRef]

5. Akgül, A.; Akgül, E.K. On solutions of new type fractional differential equations occurring in the electrohydrodynamic flow. Electron. Res. Arch. 2020, 28, 537.

6. Farman, M.; Akgül, A.; Ahmad, A.; Imtiaz, S. Analysis and dynamical behavior of fractional-order cancer model with vaccine strategy. Math. Methods Appl. Sci. 2020, 43, 4871-4882. [CrossRef]

7. Modanli, M.; Akgül, A. On Solutions of Fractional order Telegraph partial differential equation by Crank-Nicholson finite difference method. Appl. Math. Nonlinear Sci. 2020, 5, 163-170. [CrossRef]

8. Nagle, R.K.; Staff, E.B.; Snider, A.D. Fundamentals Dfferential Equations; Pearson: Upper Saddle River, NJ, USA, 2008.

9. Baleanu, D.; Fernandez, A.; Akgül, A. On a fractional operator combining proportional and classical differintegrals. Mathematics 2020, 8, 360. [CrossRef]

10. Gupta, S.; Kumar, D.; Singh, J. Analytical solutions of convection-diffusion problems by combining Laplace transform method and homotopy perturbation method. Alexasandria Eng. J. 2015, 54, 645-651. [CrossRef]

11. Anjum, N.; He, J.H. Laplace transform, making the variational iteration method easier. Appl. Math. Lett. 2019, 92, 134-138. [CrossRef] 
12. Zhou, Z.; Ma, J.; Gao, X. Convergence of iterative Laplace transform methods for a system of fractional PDEs and PIDEs arising in option pricing. East Asian J. Appl. Math. 2018, 8, 782-808. [CrossRef]

13. Bashir, T.; Kalim, M. Solution of non-homogeneous differential equations using faddeev-leverrier method together with Laplace transform. Adv. Differ. Equations Control. Process. 2018, 19, 343-357. [CrossRef]

14. Jingtang, M.; Zhiqiang, Z. Convergence analysis of iterative Laplace transform methods for the coupled PDEs from regime-switching option pricing. J. Sci. Comput. 2018, 75, 1656-1674.

15. Eljaoui, E.; Melliani, S.; Chadli, L.S. Aumann fuzzy improper integral and its application to solve fuzzy integro-differential equations by Laplace transform method. Adv. Fuzzy Syst. 2018, 2018, 9730502. [CrossRef]

16. Zhou, Z.; Ma, J.; Sun, H.W. Fast Laplace transform methods for free-boundary problems of fractional diffusion equations. J. Sci. Comput. 2018, 74, 49-69. [CrossRef]

17. Yonghong, S.; Wei, C. Laplace Transform method for the ulam stability of linear fractional differential equations with constant coefficient. Mediterr. J. Math. 2017, 14, UNSP 25.

18. Fatoorehchi, H.; Abolghasemi, H. Series solution of nonlinear differential equations by a novel extension of the Laplace transform method. Int. J. Comput. Math. 2016, 93, 1299-1319. [CrossRef]

19. Jacobs, A.B. High-order compact finite difference and Laplace transform method for the solution of time-fractional heat equations with Dirichlet and Neumann boundary conditions. Numer. Methods Partial. Differ. Equ. 2016, 32, 1184-1199. [CrossRef]

20. Gao, W.; Veeresha, P.; Prakasha, D.G.; Baskonus, H.M. Novel dynamical structures of 2019-nCoV with nonlocal operator via powerful computational technique. Biology 2020, 9, 107. [CrossRef]

21. Sulaiman, T.A.; Bulut, H.; Atas, S.S. Optical solitons to the fractional Schrödinger-Hirota equation. Appl. Math. Nonlinear Sci. 2019, 4, 535-542. [CrossRef]

22. Özarslan, M.A. On a singular integral equation including a set of multivariate polynomials suggested by Laguerre polynomials. Appl. Math. Comput. 2014, 229, 350-358. [CrossRef]

23. Kürt, C.; Özarslan, M.A.; Fernandez, A. On a certain bivariate Mittag-Leffler function analysed from a fractional-calculus point of view. Math. Meth. Appl. Sci. 2020. [CrossRef]

24. Logeswari, K.; Ravichandran, C. A new exploration on existence of fractional neutral integro- differential equations in the concept of Atangana-Baleanu derivative. Phys. A Stat. Mech. Its Appl. 2020, 544, 123454. [CrossRef]

25. Ravichandran, C.; Logeswaria, K.; Jarad, F. New results on existence in the framework of Atangana-Baleanu derivative for fractional integro-differential equations. Chaos Solitons Fractals 2019, 125, 194-200. [CrossRef]

26. Kumar, S.; Nisar, K.S.; Kumar, R.; Cattani, C.; Samet, B. A new Rabotnov fractional-exponential function-based fractional derivative for diffusion equation under external force. Math. Methods Appl. Sci. 2020, 43, 4460-4471. [CrossRef]

27. Kumar, S.; Kumar, A.; Odibat, Z.; Aldhaifallah, M. Kottakkaran Sooppy Nisar, A comparison study of two modified analytical approach for the solution of nonlinear fractional shallow water equations in fluid flow. AIMS Math. 2020, 5, 3035-3055. [CrossRef]

28. Manafianheris, J. Solving the integro-differential equations using the modified Laplace Adomian decomposition method. J. Math. Ext. 2012, 6, 1-15.

29. Manafianheris, J.; Lakestani, M. New Improvement of the Expansion Methods for Solving the Generalized Fitzhugh-Nagumo Equation with Time-Dependent Coefficients. Int. J. Eng. Math. 2015, 2015, 107978.

30. Acay, B.; Baş, E.; Abdeljawad, T. Fractional economic models based on market equilibrium in the frame of different type kernels. Chaos Solitons Fractals 2020, 130, 109438. [CrossRef]

31. Gao, W.; Veeresha, P.; Baskonus, H.M.; Prakasha, D.G.; Kumar, P. A New Study of Unreported Cases of 2019-nCOV Epidemic Outbreaks. Chaos Solitons Fractals 2020, 138, 1-6. [CrossRef]

32. Cattani, C. A review on Harmonic Wavelets and their fractional extension. J. Adv. Eng. Comput. 2018, 2, 224-238. [CrossRef]

33. Cattani, C.; Rushchitskii, Y.Y. Cubically nonlinear elastic waves: Wave equations and methods of analysis. Int. Appl. Mech. 2003, 39, 1115-1145. [CrossRef] 
34. Podlubny, I. Fractional Differential Equations; Academic Press: San Diego, CA, USA, 1999.

35. Fernandez, A.; Kürt, C.; Özarslan, M.A. A naturally emerging bivariate Mittag-Leffler function and associated fractional-calculus operators. arXiv 2020, arXiv:2002.12171.

(c)

(C) 2020 by the authors. Licensee MDPI, Basel, Switzerland. This article is an open access article distributed under the terms and conditions of the Creative Commons Attribution (CC BY) license (http:/ / creativecommons.org/licenses/by/4.0/). 